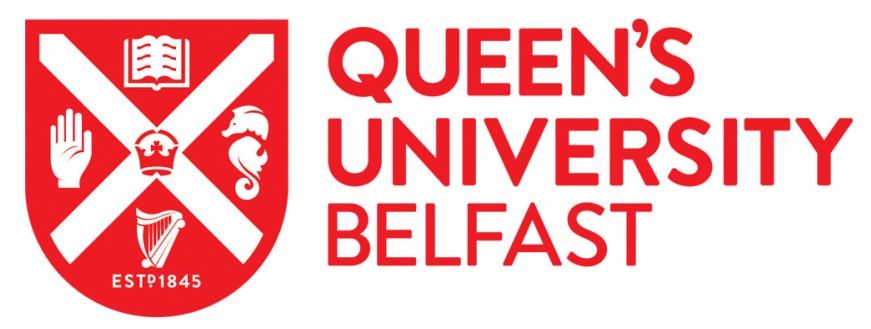

\title{
Applications and Developments in Targeted Proteomics: From SRM to DIAVSWATH
}

Aebersold, R., Bensimon, A., Collins, B. C., Ludwig, C., \& Sabido, E. (2016). Applications and Developments in Targeted Proteomics: From SRM to DIA/SWATH. Proteomics, 16(15-16), 2065-2067.

https://doi.org/10.1002/pmic.201600203

\section{Published in:}

Proteomics

\section{Document Version:}

Peer reviewed version

Queen's University Belfast - Research Portal:

Link to publication record in Queen's University Belfast Research Portal

Publisher rights

Copyright 2016 Wiley. This work is made available online in accordance with the publisher's policies. Please refer to any applicable terms of use of the publisher.

\section{General rights}

Copyright for the publications made accessible via the Queen's University Belfast Research Portal is retained by the author(s) and / or other copyright owners and it is a condition of accessing these publications that users recognise and abide by the legal requirements associated with these rights.

Take down policy

The Research Portal is Queen's institutional repository that provides access to Queen's research output. Every effort has been made to ensure that content in the Research Portal does not infringe any person's rights, or applicable UK laws. If you discover content in the Research Portal that you believe breaches copyright or violates any law, please contact openaccess@qub.ac.uk. 


\section{Editorial for special issue 'Applications and developments in targeted proteomics: from SRM to DIA/SWATH'}

The adaptation of targeted data acquisition from small molecule analysis to bottom-up proteomics in the form of selected reaction monitoring (SRM) approximately a decade ago was initially motivated by the need for robust and sensitive quantification of proteins in both basic life science research and translational medicine. This need was precipitated by limitations with respect to quantification, repeatability and reproducibility that were associated with data dependent acquisition (DDA) caused by the now well described issues surrounding stochasticity of precursor selection ${ }^{1}$. Over the years, the capabilities of targeted data acquisition by $\mathrm{SRM}^{2}$, and its more recently implemented cousin parallel reaction monitoring 3,4 (PRM), have developed remarkably in both instrumentation and software, enabling robust and sensitive quantification of discrete sets of proteins across large sets of samples. Further, the quality of the quantitative data available from targeted approaches and the robustness of the methods have enabled highly successful applications in a variety of arenas and this has led to broad community acceptance of the utility of targeted proteomics ${ }^{5}$.

While targeted data acquisition by SRM or PRM has been successful, the methods are also limited in utility for some applications. Most obviously, data is acquired for a set of proteins, defined prior to acquisition and once acquired, there is no possibility to re-mine the data to obtain information for other targets. In principle, data independent acquisition ${ }^{6}$ (DIA) alleviated the requirement to define a set of proteins prior to acquisition and could expand to quantify larger sets of proteins. The introduction of targeted data analysis to DIA, implemented as SWATH-MS ${ }^{7}$, drew on analysis principles similar to those developed for data from targeted acquisition. This marked a turning point in DIA that facilitated the generation of data that maintained the favorable quantitative characteristics of targeted data acquisition, while significantly expanding the coverage of proteins compared to SRM or PRM. This advance has led to significant method developments in this space, in parallel to successful applications in many research areas.

The term DIA has broadly referred to a range of deterministic acquisition methods ${ }^{8}$ accompanied by data analysis strategies ${ }^{9}$ which fall into either the peptide-centric analysis category ${ }^{10,11}$, utilizing targeted data analysis similar to SWATH-MS, or the spectrum-centric data analysis category, that are conceptually more similar to classical database search strategies. To capture the range of approaches, we have chosen to use the inclusive term DIA/SWATH in the title of this special issue to indicate that the focus here is on DIA strategies which draw on the concepts and analysis strategies which are characteristic of targeted proteomics (as in SWATH-MS). Furthermore, applications in DIA/SWATH may use terms borrowed from targeted data acquisition such as "targeted assay" or "library" to describe a set of analyte parameters used for data extraction from DIA data. Additional discussion could examine and perhaps refine these terms in the context of DIA, for example, similar to a recent community effort which proposed categorization of assays of various stringency levels for different types of basic science or clinical applications in targeted MS measurements ${ }^{12}$. We hope this special issue will stimulate such a conversation in this area regarding terminology.

The goal of this special issue was to sample the breadth of applications currently being pursued using these techniques as well as to represent ongoing technical developments. In the first section of the volume, 7 papers describe applications in the scope of the special issue. In the second section, 6 papers review and compare different techniques and discuss differences between them. And in the third and final section, 5 papers suggest various developments in acquisition and data analysis.

With these applications and developments in mind we might ask what will the future hold for targeted proteomics and related DIA-based methods? With the rapid increase in the scanning speed 
of high resolution mass spectrometers the lines between approaches such as PRM, DDA, and DIA/SWATH will begin to blur and a unified acquisition method could emerge in which high resolution MS2 spectra are acquired deterministically with narrow precursor isolation windows and comprehensive sampling of the precursor space. With such comprehensive and high quality data it seems likely that the focus of discussion will be whether the data should be analyzed by peptidecentric approaches or by spectrum-centric approaches. With the availability of unprecedented data sets generated by these methods, the focus of quantitative proteomics might also partially shift away from technical aspects towards the most innovative applications of the powerful technology to generate new biological and clinical knowledge.

What then is the future for targeted proteomics using low resolution mass spectrometers (e.g SRM)? The sensitivity, relatively low cost and robustness of triple quadrupoles will likely keep this method in favor for routine applications where discrete sets of proteins need to be measured in large numbers of samples. In particular, translating targeted assays into analytical tier 1 assays for potential future applications in clinical research remains an attractive prospect ${ }^{12}$. In broader terms, targeted proteomics using SRM/PRM for basic research purposes is also likely to persist as the level of sensitivity and selectivity achievable may be still favorable compared to DIA-based methods.

We would like to thank all of the authors who contributed articles to this special issue, the reviewers who provided critical feedback, and the editors of PROTEOMICS for making this special issue possible. Based on the enthusiastic response to the call for submissions to this special issue, one point is clear: The interest in continuing to develop and apply methods in this area is increasing and there are surely exciting times ahead. We speculate that the bright line that has separated targeted proteomics from discovery proteomics will begin to fade leaving only a broad quantitative proteomics method for life science research which is robust, reproducible and comprehensive ${ }^{13}$. We hope that the collection of papers in this special issue will help to underscore the power and potential of this arena of quantitative proteomics.

Guest editors: Ruedi Aebersold, Ariel Bensimon, Ben C. Collins, Christina Ludwig, Eduard Sabido

1. Tabb, D. L. et al. Repeatability and Reproducibility in Proteomic Identifications by Liquid Chromatography-Tandem Mass Spectrometry. J. Proteome Res. 9, 761-776 (2010).

2. Lange, V., Picotti, P., Domon, B. \& Aebersold, R. Selected reaction monitoring for quantitative proteomics: a tutorial. Mol. Syst. Biol. 4, 222 (2008).

3. Peterson, A. C., Russell, J. D., Bailey, D. J., Westphall, M. S. \& Coon, J. J. Parallel Reaction Monitoring for High Resolution and High Mass Accuracy Quantitative, Targeted Proteomics. Mol. Cell. Proteomics 11, 1475-1488 (2012).

4. Gallien, S., Bourmaud, A., Kim, S. Y. \& Domon, B. Technical considerations for large-scale parallel reaction monitoring analysis. J. Proteomics 100, 147-159 (2014).

5. Method of the Year 2012. Nat. Methods 10, 1-1 (2013).

6. Venable, J. D., Dong, M.-Q., Wohlschlegel, J., Dillin, A. \& Yates, J. R. Automated approach for quantitative analysis of complex peptide mixtures from tandem mass spectra. Nat. Methods $\mathbf{1}$, 39-45 (2004).

7. Gillet, L. C. et al. Targeted data extraction of the MS/MS spectra generated by data-independent acquisition: a new concept for consistent and accurate proteome analysis. Mol. Cell. Proteomics MCP 11, 0111.016717 (2012).

8. Chapman, J. D., Goodlett, D. R. \& Masselon, C. D. Multiplexed and data-independent tandem mass spectrometry for global proteome profiling. Mass Spectrom Rev 33, 452-70 (2013). 
9. Bilbao, A. et al. Processing strategies and software solutions for data-independent acquisition in mass spectrometry. PROTEOMICS 15, 964-980 (2015).

10. Ting, Y. S. et al. Peptide-Centric Proteome Analysis: An Alternative Strategy for the Analysis of Tandem Mass Spectrometry Data. Mol. Cell. Proteomics 14, 2301-2307 (2015).

11. Gillet, L. C., Leitner, A. \& Aebersold, R. Mass Spectrometry Applied to Bottom-Up Proteomics: Entering the High-Throughput Era for Hypothesis Testing. Annu. Rev. Anal. Chem. 9, (2016).

12. Carr, S. A. et al. Targeted Peptide Measurements in Biology and Medicine: Best Practices for Mass Spectrometry-based Assay Development Using a Fit-for-Purpose Approach. Mol. Cell. Proteomics 13, 907-917 (2014).

13. Nilsson, T. et al. Mass spectrometry in high-throughput proteomics: ready for the big time. Nat. Methods 7, 681-685 (2010). 